\title{
Percepção das mulheres no município de Paulo Afonso, na Bahia, sobre as mudanças corporais e emocionais no período do climatério
}

\author{
Árquisa Antônia de Sousa Santos ${ }^{1}$, Flávia Ventura da Silva² e \\ Fabiana Lopes Martins ${ }^{3}$
}

\footnotetext{
1 Especialização em Urgência e Emergência pela Especializa Saúde em Campina Grande-PB. Graduação em enfermagem pela União de Ensino Superior de Campina Grande. Atualmente é Preceptora da Faculdade de Ciências Humanas e Sociais (AGES), Brasil. Email: arquisasousa@hotmail.com

2 Graduada em Enfermagem pela Faculdade de Ciência Humanas e Socias-AGES, Brasil. E-mail: flaviaventura2010@hotmail.com

3 Especialista em Enfermagem do Trabalho pela Universidade Católica Dom Bosco (UCDB) e Educação Profissional na Área da Saúde pela Universidade Federal de Minas Gerais (UFMG). Graduada em Enfermagem pela Faculdade de Enfermagem e Obstetrícia de Jaú, Brasil.
}

RESUMO: Este estudo teve como objetivo analisar a percepção de mulheres assistidas em uma unidade de Estratégia de Saúde da Família localizada no município de Paulo Afonso, Bahia, acerca das mudanças corporais e emocionais no climatério. Trata-se de uma pesquisa exploratória, com abordagem quanti-qualitativa. A amostra foi constituída por 30 mulheres. As variáveis quantitativas foram analisadas por distribuição de porcentagem e comparadas à luz da literatura atual. Os dados qualitativos foram analisados através da análise temática de conteúdo proposta por Bardin (2004). Os resultados evidenciaram que $100 \%$ das entrevistadas afirmaram não conhecer o termo climatério; $66,6 \%$ disseram que os primeiros sintomas desta fase sugiram entre 41 e 50 anos; entre os sintomas, $86,6 \%$ citam as ondas de calor. A análise qualitativa revelou que as mulheres consideram a fase como um período difícil e desagradável em virtude das alterações físicas e psicológicas. Concluiu-se que o climatério é um período importante da vida da mulher e que para a maioria das mulheres deste estudo o climatério é um período marcado por mudanças negativas.

Palavras-chave: Climatério. Percepção. Enfermagem. Saúde da Mulher.

Perception of women in Paulo Afonso, in Bahia, on the physical and emotional changes in the climacteric period

ABSTRACT: This study aimed to analyze the perception of bodily and emotional changes women on perimenopause assisted in a unit of the Family Health Strategy in the municipality of Paulo Afonso, Bahia. This is a field research, exploratory, with quantitative and qualitative approach. The sample consisted of 30 women. Quantitative variables were analyzed by distribution percentages and compared in the light of current literature. Qualitative data were analyzed using thematic content analysis proposed by Bardin (2004). The results showed that $56.7 \%$ of respondents were aged between 41 and 50 years; 30\% stopped menstrual cycle course for over 4 years; $100 \%$ say they do not know the term menopause. $66.6 \%$ of respondents said that the first symptoms of this stage suggest between 41 and 50 , 
among the symptoms $86.6 \%$ of women cite hot flashes. Qualitative analysis revealed that women consider the stage as a difficult and unpleasant period because of the physical and psychological changes. It was concluded that menopause is an important period of life of women and that for most women in this study perimenopause is a period marked by negative changes.

Keywords: Climacteric. Perception.Nursing.Women's Health.

\section{INTRODUÇÃO}

Nos últimos anos, houve um aumento da expectativa de vida das mulheres, consequência da evolução dos procedimentos e tratamentos médicos e da melhoria da qualidade de vida. Em nosso país, as mulheres representam a maioria da população idosa e vivem, em média, oito anos mais do que os homens. Com isso, elas passaram a viver tempo suficiente para vivenciar modificações que muitas gerações anteriores não tiveram a oportunidade de alcançar (ARAÚJO, 2009).

Um dos acontecimentos percebidos na vida das mulheres que alcançam a longevidade é o climatério e, segundo Araúio (2009), a maior expectativa de vida das muIheres aumentou o número de estudos e pesquisas com relação a essa nova fase de vida, até então desconhecida.

O climatério é um período em que a função dos ovários diminui de forma gradativa até cessar completamente, fase de transição na vida da mulher que marca a passagem da fase reprodutiva da fase não reprodutiva (BRASIL, 2008). Esse período biológico da vida feminina é composto por um conjunto de alterações orgânicas e emocionais, cujo início se confunde com o final do período reprodutivo. $O$ termo climatério provém da palavra grega "climarkter" que significa "ponto crítico da vida humana" (ALMEIDA, 2003).
O climatério não representa uma doença, mas é caracterizado como um estado de crescente carência estrogênica e pelos prodígios do envelhecimento. No entanto, esse binômio, quando não bem acompanhado, pode originar processos patológicos (BRASIL, 2008). Na maioria das mulheres, esse período ocorre em torno dos 35 anos e se estende até os 65 , período em que a muIher pode apresentar alterações clínicas que podem interferir no seu estado de saúde; algumas dessas alterações geralmente levam a síndrome climatérica mais frequente, no período da peri-menopausa, ou seja, após os 45 anos de idade (BRASIL, 2008).

De acordo com Pinto (2006), a sintomatologia climatérica atinge cerca de $85 \%$ das mulheres acima dessa faixa etária e no Brasil tem aumentado consideravelmente, a prevalência dela em mulheres que experimentam, no mínimo, um dos sintomas climatéricos no pais é cerca de $96,9 \%$.

Com todas essas modificações as mulheres procuram a melhor forma de harmonizar-se nessa fase. Para auxilia-las, o Ministério da Saúde, em 2008, lançou o Manual de Atenção Integral à Saúde da Mulher no Climatério/Menopausa, para nortear às ações voltadas a saúde da mulher climatérica (BRASIL, 2008). Atualmente, a atenção básica de saúde, sobretudo a Estratégia Saúde da Família (ESF) tem dado ênfase no tratamento das mulheres climatéricas. 
Para Araújo (2009), o climatério refere-se ao período anterior e ao posterior à menopausa, marcado por sinais de deficiência do ovário em produzir hormônios. No período de climatério as mulheres apresentam taxas hormonais distintas das taxas apresentadas por mulheres fora desse período. Há um decréscimo na produção de estradiol e uma elevação dos níveis dos hormônios folículoestimulante (FSH) e luteinizante (LH) que acabam por influenciar mudanças em todo organismo.

Eventualmente, o período do climatério pode ser assintomático em aproximadamente $25 \%$ das mulheres, no entanto, no restante, podem surgir sintomas que caracterizam a síndrome climatérica (CAMPANA; NETO; PEDRO, 2001). A presença de sinais e sintomas no climatério difere de mulher para mulher, geralmente, surgem a partir dos 40 anos, ou podem começar, tardiamente, após os 50 anos. De acordo com De Lorenzi et al. (2005), aproximadamente, $70 \%$ das mulheres relatam sintomas somáticos e problemas emocionais nos anos que seguem este período.

O estresse, a depressão e o consumo de produtos tóxicos, como cigarros e álcool, além de cirurgias ginecológicas, como a histerectomia, podem ser considerados como principais responsáveis por um climatério sintomatológico, ou seja, a presença de sintomas, assim como a magnitude desses está diretamente relacionada com a visão de mundo, a história de vida, dos hábitos, vivências e experiências das mulheres (ARAÚJO, 2009).

Conforme Santos (2007) as principais alterações clínicas são: neurogênicas, psicogênicas, metabólicas, mamárias, urogenitais, ósteo-articulares e do sistema tegumentar (pele e anexos).
O diagnóstico do climáterio é fundamentalmente clínico, elaborado a partir dos sintomas descritos anteriormente, entretanto em algumas situações, a critério do médico, pode ser realizada a solicitação de exames complementares nesse período com função preventiva, e alguns podem auxiliar o diagnóstico em casos atípicos (BIFFI, 2003).

Ainda segundo Biffi (2003), a avaliação clinica para constatar esse acontecimento (climáterio) deve ser realizada em três etapas: anamenese, exame físico detalhado e exames laboratoriais, sendo importante considerar o climáterio, do ponto de vista orgânico e psíquico. A confirmação do diagnóstico pode ser feita pelas dosagens de FSH e LH que estão aumentados e de estradiol que se encontra diminuído.

Existem diversas alternativas para redução dos sintomas que surgem durante o climatério, dentre eles, a terapia com reposição hormonal (TRH), realizada principalmente com estrógenos e progesteronas a sua associação tem indicação no controle ou na redução das manifestações vasomotores e urogenitais decorrentes da redução da produção de esteroides ovarianos em especial a progesterona e o estradiol (WANNMACHER; LUBIANCA, 2004).

Este estudo teve como objetivo analisar a percepção acerca das mudanças corporais e emocionais das mulheres sobre o climatério assistidas na Estratégia de Saúde da Família de São João, localizada no município de Paulo Afonso, Bahia.

\section{MÉTODO}

Para o alcance dos objetivos deste trabaIho, optamos pela realização de estudo exploratório com abordagem quanti-qualitativa. Segundo Prestes (2003) a pesquisa ex- 
ploratória segundo tem por objetivo proporcionar maiores informações sobre o assunto a ser investigado, facilitando a delimitação do tema, orientando a delimitação dos objetivos e a formação de possíveis hipóteses ou descobrir uma nova possibilidade de enfoque para o assunto. Fortin (2009) afirma que a investigação quantitativa, visa, principalmente explicar e predizer um fenômeno pela medida das variáveis e pela análise de dados numéricos e que a investigação qualitativa tem por objetivo a compreensão alargada dos fenômenos.

A pesquisa foi realizada na Estratégia de Saúde da Família do município de Paulo Afonso, Bahia, haja vista a sua importância para a comunidade. A pesquisa realizou-se nos turnos matutino e vespertino. Durante o seu desenvolvimento, a ESF contava com a seguinte equipe: um médico clínico geral; uma enfermeira; duas técnicas de enfermagem; cinco agentes comunitários de saúde; um motorista, uma auxiliar de serviços gerais, três vigilantes, um odontólogo e uma auxiliar de saúde bucal. Os participantes do estudo foram constituídos por mulheres climatéricas cadastradas e atendidas na Estratégia Saúde da Família, no município de Paulo Afonso no Estado da Bahia.

A amostra foi composta por 30 mulheres que estavam disponíveis e concordaram mediante a assinatura do Termo de Consentimento Livre e Esclarecido (TCLE). O tamanho da amostra foi definido à medida que os dados foram saturados, não havendo acréscimo à pesquisa. Como critério de inclusão para participar da pesquisa, delimitamos mulheres atendidas no turno da tarde, nos dias de quinta-feira, com faixa etária entre 35 e 60 anos, que, voluntariamente, desejassem participar do estudo, apresentassem capacidade cognitiva preserva- da, residissem na área de abrangência da $E S F$, estivessem em climatério natural e concordassem em assinar o termo de consentimento.

O instrumento escolhido foi um formulário. A entrevista foi direcionada pela questão norteadora: Qual a percepção das mulheres sobre as mudanças corporais e emocionais no período do climatério? Esta foi gravada com uso de um microgravador, após anuência das nove mulheres participantes, assinando o Termo de Consentimento Livre e Esclarecido (TCLE).

Com a finalidade de garantir o anonimato e o sigilo dos sujeitos do estudo, estabeleceu-se pseudônimos utilizando nome de flores para identificação das trinta mulheres participantes. Os dados foram coletados individualmente em uma sala reservada na unidade de saúde, no período de maio de 2015.

Após a coleta dos dados, procedeu-se a análise e interpretação dos mesmos. Os resultados foram apresentados de duas formas. Primeiro, expressamos a parte do estudo quantitativo. Por ter sido primeiramente coletado, eles foram calculados e transformados em tabelas e gráficos com o auxílio do programa Microsoft Office Excel do sistema operacional Microsoft Windows.

A seguir, apresentamos os resultados dos dados qualitativos utilizando, para isto, a análise temática de conteúdo proposta por Bardin (2004).

Os dados foram interpretados mediante análise de discurso e classificados por meio de um questionamento com base na fundamentação teórica, sendo elaboradas categorias relacionadas a um conceito central e reunidas por meio de elementos ou aspectos comuns. 
A fim de obter uma adequada análise, foram seguidos os passos: ordenação dos dados e mapeamento dos dados; classificação dos dados, que consiste na leitura exaustiva do material para identificação de temas relevantes e estabelecimento de categorias temáticas; e análise final, que é a realização de articulações entre os dados e autores pesquisados. Após a transcrição e a leitura dos relatos, foi realizada a seleção das temáticas que apareceram como as mais destacadas nas falas das informantes, ou seja, aquilo que estava sendo mais importante e problematizado, pertinente aos objetivos da pesquisa.

A aplicação do formulário se deu com esclarecimento sobre a importância do estudo e como ele seria desenvolvido, as participantes foram convidadas a concordarem com o Termo de Consentimento Livre e Esclarecido (TCLE) através de sua assinatura, em duas vias, onde uma ficou com o pesquisador e outra com a participante da pesquisa, assegurando assim o seu anonimato e a privacidade das entrevistadas.

A pesquisa foi fundamentada e delineada no que preconiza a Resolução 466/12 do Conselho Nacional de Saúde que regulamenta a pesquisa e aplica as normas necessárias ao desenvolvimento da mesma, pelo fato de envolverem, diretamente ou indiretamente, seres humanos. Inicialmente foi solicitado a autorização para a realização da pesquisa a Secretaria Municipal de Saúde do Município de Paulo Afonso, após sua autorização enviou-se o projeto para o comitê de ética da faculdade AGES. O projeto foi submetido à avaliação do Comitê de Ética em Pesquisa da Faculdade de Ciências Humanas e Sociais-AGES, que foi aprovado através do Protocolo 075-2014, somente após a aprovação do comitê procedeu-se a coleta de dados.

\section{RESULTADOS E DISCUSSÃO}

Os dados levantados através dos formulários foram avaliados e discutidos. As variáveis quantitativas foram analisadas por distribuição de porcentagem e comparadas à luz da literatura atual. Os dados qualitativos foram analisados através da técnica de análise temática de conteúdo, proposta por Bardin (2004).

A amostra foi composta por 30 mulheres, todas, usuárias da Estratégia Saúde da Família de São João. A análise estatística revelou que as mulheres participantes da pesquisa possuem idade entre 35 e 56 anos, significando que todas estão vivenciando o climatério.

Destas mulheres, $16,7 \%(n=5)$ se situaram entre 35 e 40 anos; $56,7 \%(n=17)$ entre 41 e 50 anos e $26,6 \%(n=8)$ entre 51 e 60 anos. A média de idade foi de 45,7 anos. Este estudo diferenciou-se de outros realizados, por apresentar uma média de idade inferior a estudos efetuados em outras regiões do Brasil, onde a média de faixa etária está entre 53,7; 52,3; 54 e 51,4 anos (DE LORENZI et al., 2005; VIDAL, 2009).

Ao serem questionadas sobre seu estado civil constatou-se que a maioria das mulheres deste estudo $63,3 \%(n=19)$ era casadas e vivia com o esposo; apenas $3,4 \%(n=1)$ se declarou solteira e $33,3 \%(n=10)$ divorciadas.

Fonseca et al (1999) apontam que muIheres climatéricas casadas referem maior grau de insatisfação sexual do que as amasiadas e separadas com atividade sexual.

Em relação ao perfil das participantes, a maioria, $33,3 \%(n=10)$ não era alfabetizada, 
$23,3 \%(n=7)$ possuía somente o ensino fundamental incompleto $16,7 \%(n=5)$ o ensino fundamental completo, $16,7 \%(n=5)$ o ensino médio incompleto e apenas $10 \%(n=3)$ destas mulheres chegaram a concluir o ensino médio. Este estudo mostrou mulheres com baixo grau de instrução relacionadas com maior número de sintomas decorrentes do período do climatério. Segundo Souza e Fioravente (2008), o nível educacional da mulher pode influenciar em suas atitudes preventivas, através da melhor compreensão das informações sobre as doenças e da necessidade de atitudes favoráveis à deteç̧ão precoce das neoplasias. Portanto, durante a consulta ginecológica de enfermagem, a enfermeira deve enfatizar a importância da educação em saúde, realizando as mais diversas orientações. Já que, de acordo com Moura et al. (2010), a baixa escolaridade das mulheres dificulta o desenvolvimento das ações de saúde, devido à má compreensão dessas orientações.

Quantos aos dados ginecológicos, 6,6\% $(n=2)$ informaram que a sua primeira menstruação ocorreu com 12 anos de idade; $36,6 \%(n=11)$ disseram que a menarca ocorreu aos 13 anos; a maioria 53,4\% ( $n=16)$ informou que a menarca foi aos 14 anos e apenas $3,4 \% \quad(n=1)$ disse que a primeira menstruação ocorreu aos 18 anos. Conforme Roman et al. (2009) a idade média para menarca no Brasil é de 12,2 anos. No estudo não se observou nenhuma relação entre a idade da menarca e os sintomas do climatério.

Quando questionadas quanto à menopausa $30 \%(n=9)$ responderam ter cessado o ciclo menstrual naturalmente há mais de 04 anos e $70 \%(n=21)$ tinham fluxo menstrual, algumas das participantes disseram apresentar o fluxo menstrual com irregula- ridade. A menopausa, última menstruação da mulher é reconhecida após doze meses ou mais de amenorreia. Ela ocorre em consequência da redução na secreção hormônios ovarianos o estradiol e progesterona devido, à perda da função folicular ovariana. No Brasil, a idade para a menopausa de causa natural varia entre 48 e 50 anos (BRASIL, 2008).

Em relação a vida sexual, $80 \%(n=24)$ das mulheres abordadas têm vida sexual ativa e apenas $20 \%(n=6)$ relatam não ter vida sexual ativa. Aquelas que responderam manter relações sexuais, o fazem, mas sem a mesma frequência de quando mais jovens. A diminuição da libido, evidenciada por algumas mulheres, segundo Praxedes (2011) pode ser atribuída às modificações anatômicas e funcionais decorrentes da redução estrogênica. Os tecidos vaginais tornam-se mais finos. Ocorre diminuição da elasticidade e da lubrificação. Com isso, as relações sexuais tornam-se desconfortáveis, desencadeando, em alguns casos, a diminuição do desejo sexual.

Sobre os resultados relativos à análise do hábito de vida, $83,3 \%(n=25)$ não fumavam e apenas $16,7 \%(n=5)$, conforme apresentado na Tabela 5.3, era tabagista. Verificou-se no estudo que, entre as mulheres fumantes, os fogachos estão presentes com mais frequências e intensidade. Este resultado é semelhante aos resultados encontrados por Sclowitz, Santos e Silveira (2005) sobre prevalência e fatores associados a fogachos em mulheres climatéricas e pós-climatéricas, onde constataram que as mulheres fumantes relatavam fogachos mais frequentemente $(36,7 \%)$ do que as ex-fumantes $(31,9 \%)$ e as que nunca fumaram $(26,4 \%)$.

No que diz respeito à atividade física, apenas $30 \%(n=9)$ das participantes relata- 
ram praticar alguma atividade física regularmente. Entre as atividades realizadas, encontra-se, principalmente, a caminhada e a maioria $70 \%(n=21)$ assumiu ser sedentária, justificada pela falta de tempo hábil para realizar uma atividade física. Todavia, Praxedes (2011) aponta em seu estudo que mulheres climatéricas sedentárias têm maior chance de apresentar sintomas do climatério quando comparadas àquelas com relato de prática frequente de exercícios físicos.

Sobre a percepção das mulheres quanto o climatério, inicialmente, é necessário enfatizar que ao perguntar sobre o significado do climatério $100 \%(n=30)$ das participantes não conheciam o termo e nunca tinham ouvido falar sobre o assunto, por isso, para obter informação a respeito do assunto foi preciso esclarecer a elas o significado da referida expressão. Logo associaram o climatério à menopausa e revelaram o conhecimento que possuíam sobre o mesmo e percepções sobre o período vivido por elas.

Nota-se que as participantes da pesquisa não possuem o conhecimento devido sobre o climatério, em consequência disto, poderão sofrer com as alterações fisiológicas e principalmente as psicológicas, proporcionando um estado emocional debilitado, trazendo inseguranças e desmotivações devido o novo período de transformações em suas vidas tornando este momento bastante desagradável.

No estudo foi observado que a maioria das mulheres entrevistadas mostrou interesse em conhecer o que era o climatério e as causas das alterações pelas quais estão passando, evidenciando, assim, a desinformação da população feminina sobre o climatério, fase da vida das mulheres marcadas por profundas alterações que requer das mesmas, certo conhecimento para en- frentar este período com maturidade e adaptação.

Este resultado vai a encontro com os de Milanez e Nery (2004) que para obter as informações das mulheres em seu estudo sobre percepção das mulheres sobre o climatério: bases para a assistência de enfermagem, realizado em Teresina, no Piauí, tiveram que explicar o termo climatério para as entrevistadas.

Segundo Gonçalves e Merighi (2007), o climatério é um fenômeno bio-psicossocial que representa a fase de transição entre a menacme (período compreendido entre a menarca e a menopausa) e a senectude, e abrange a transição do estágio reprodutor para o não reprodutor.

No que diz respeito à idade em que se iniciaram os primeiros sintomas do climatério, $66,6 \%(n=20)$ das mulheres apresentaram os primeiros sintomas entre 41 e 50 anos de idade, $20 \%(n=6)$ relatam entre 51 e 60 anos e apenas $13,4 \%(n=4)$ antes dos 40 anos de idade. Estes dados revelam compatibilidade com as literaturas que dizem que os primeiros sintomas surgem no período de peri-menopausa, período este, situado segundo Praxedes (2011) entre 41 e 51 anos.

Quanto aos sintomas referidos no período do climatério, diversos sintomas incomodavam e interferiam nas atividades diárias, repercutindo no seu comportamento social, familiar e profissional. Destas, $86,6 \%$ $(n=26)$ citaram as ondas de calor como um problema a ser enfrentado no período do climatério e apenas $13,4 \%(n=4)$ das pesquisadas disseram não senti-los durante esta fase. Estes dados revelam a alta incidência dos fogachos nas mulheres entrevistadas.

Segundo Cruz (2009) os fogachos são ocasionados provavelmente pela queda dos 
níveis de estradiol, que geralmente causam impactos negativos na qualidade de vida das mulheres, podendo desencadear desconforto físico agudo, tanto quanto distúrbios do sono, o que resulta em fadiga, irritabilidade e até condições clinicas sérias como a depressão.

Os dados a seguir descrevem a prevalência da cefaleia, onde $60 \%(n=18)$ referiram à presença atual do sintoma. Das 30 mulheres apenas 40\% (n=12) não apresentavam. Estes dados estão de acordo com um estudo sobre sintomatologia climatérica realizado por De Lorenzi et al. (2005), onde a cefaleia foi referida por $64,1 \%$ das mulheres.Dentre as mulheres estudadas, apenas $20 \%(n=6)$ apresentavam artralgia ou mialgia e maioria $80 \%(n=24)$ disse não apresentar estes sintomas.

Outro sintoma vivenciado pelas mulheres foi a insônia, relatada por $40 \%(n=12)$ das entrevistadas. A maioria das mulheres que relataram este sintoma já tinha passado pela menopausa. Estes dados vão ao encontro com os estudos realizados por Campos et al. (2005), os quais afirmam que a insônia afeta cerca de $60 \%$ das mulheres durante o climatério, principalmente após a menopausa. É interessante ressaltar que $80 \%$ $(n=24)$ das mulheres apresentam ansiedade ou irritabilidade. Percebe-se que este resultado é bem expressivo e divergem do estudo realizado por Praxedes (2011) com muIheres climatéricas, onde apenas 35\% das mulheres referiram irritabilidade com a passagem pelo climatério. Observou-se no estudo que as mudanças físicas significam muito para as mulheres, porém as mudanças emocionais costumam ter um significado especial por adentrar o campo dos sentimentos.
Quanto à fadiga, foi relatada por $73,4 \%$ $(n=22)$ das mulheres. Para relatar à fadiga as mulheres participantes deste estudo se expressaram de diversas formas, utilizando termos como: cansada, esgotada, acabada e lenta. Segundo Praxedes (2011), a fadiga está associada com fatores físicos, psicológicos, sociais, cognitivos e comportamentais.

Independentemente da mudança ter caráter físico, emocional ou misto, a mulher necessita ser ouvida, ser compreendida e ser orientada quanto a estas mudanças, cabendo os enfermeiros da Estratégia Saúde da Família, por estarem mais próximos destas mulheres, desenvolverem consultas de enfermagem com ênfase na escuta e nas ações educativas.

De Lorenzi et al. (2005) consideram que são inúmeras as possibilidades de intervenção no climatério, o que irá depender de uma escuta adequada das queixas de saúde dessas mulheres, dos seus sentimentos e percepções acerca do seu envelhecimento. Desse modo, é imprescindível que a mulher tenha espaço para expressar os sentimentos relativos ao período que está vivenciando e as dificuldades que está sentindo, de modo a receber informações sobre as modificações corporais que está sofrendo e as suas implicações para a saúde.

O estímulo à adoção de hábitos saudáveis que possam envolver uma melhoria da qualidade de vida e promover o alívio da sintomatologia climatérica inclui a mudança quanto aos hábitos alimentares, prática regular de atividade física, prescrição adequada de fármacos para redução de sintomas desagradáveis, tais como fogachos e secura vaginal, estímulo a atividades grupais de lazer e recreação, bem como apoio 
psicológico para a elevação da autoestima e alcance do bem-estar.

Para os itens subjetivos do formulário escolhemos utilizar a análise temática de conteúdo proposta por Bardin (2004). Inicialmente realizamos uma leitura do material e os fichamentos, em seguida outra leitura e a codificação do material separando o conteúdo por categorias temáticas. Foram identificadas quatro categorias: maneira como as mulheres vivenciam o climatério; sexualidade e o climatério; tratamentos utilizados para o climatério e assistência de enfermagem à saúde da mulher durante o climatério.

\subsection{Maneira como as mulheres vivenciam o climatério}

O climatério caracteriza-se como uma fase de transformações sociais, fisiológicas e psicológicas. Estas alterações são decorrentes do hipoestrogenismo que dá origem a manifestações clínicas que interferem sobre maneira que as mulheres vivenciam a vida. Portanto, constitui-se em um momento de grande vulnerabilidade para a mulher.

$\mathrm{Na}$ fala destas depoentes, podemos observar como elas se sentem nesta fase da vida. Em seus depoimentos elas citam:

é uma fase da vida da gente difícil, mas temos que passar por ela é a vida... é um momento que estamos ficando velha, surge um monte de coisa, tenho medo... o corpo da gente fica mais cansado... a gente para de menstruar e menstruar segundo minha mãe dizia é bom porque o sangue está circulando, agora vivo com dor na cabeça, nem gosto de sair de casa pois tem vezes que sinto uns suores, calores esquisitos, até parece que estou pegando fogo (ROSA).
O climatério foi considerado pelas participantes como um período difícil e desagradável em virtude das alterações físicas e psicológicas. Percebemos que as mulheres vivenciam esta experiência com certo medo, visto que elas relataram diversas mudanças que tiveram impactos negativos para a sua vida. Entre os maiores incômodos citados pelas depoentes estão as ondas de suor e calor e as mudanças de relacionamento com a família e no ambiente de trabalho.

Conforme Almeida (2003) a etiologia desses calores (fogachos) não é totalmente conhecida. Estudos realizados indicam que estes calores são ocasionados por fatores neuroendócrinos. A instabilidade do sistema termorregulador hipotalâmico, traduzida pela perda do controle vasomotor, seria causada pelos níveis circulantes de estrógeno em nível hipotalâmico e mediada pelos transmissores do sistema nervoso central. Dessa forma, determinaria vasodilatação periférica, elevação da temperatura cutânea e aceleração dos batimentos cardíacos, fatores que, associados, convergem com a descrição desses "calores" evidenciados pelas mulheres.

Apesar disso, a maioria delas entende o climatério como uma fase do ciclo da vida, que deve ser aceita e vivenciada, pois é inerente à condição de mulher. É um processo normal, vivido de forma diferente pelas muIheres.

\subsection{Sexualidade e o climatério}

A mulher durante o período de climatério frequentemente observa alterações na vivência da sua sexualidade. Isso pôde ser identificado no depoimento abaixo citado 
pelas participantes deste estudo, que mencionaram as seguintes modificações:

[...] depois dessa idade fiquei mais fria, sem vontade de fazer essas coisas, não tenho mais prazer, acho que é por que quando vou fazer minha vagina fica seca e dói, tenho até medo quando meu marido chega a noite fazendo carinho já pensando no que ele quer [...] (ORQUÍDEA).

Não tenho mais desejo não, acho que não gosto mais, quando faço parece que está rasgando tudo, me dá uma coisa ruim, no outro dia fico com muita dor de cabeça e dor nas pernas é ruim demais... meu marido pensa que é por que eu não gosto mais dele (AÇUCENA).

Para a mulher, as mudanças sexuais são consideradas incômodas, visto que refletem na sua relação com o companheiro e consigo mesma. As mudanças sexuais apontadas pelas participantes foram o ressecamento vaginal, que causa o desconforto no ato sexual, a dispauremia, dor no ato sexual geralmente causada tanto pela falta de lubrificação quanto pela atrofia vaginal e a diminuição da libido, provavelmente, ocasionada por não se sentirem a vontade com o companheiro.

Lima e Angelo (2001) identificaram que as mudanças ocorridas nessa nova fase da vida da mulher influenciam no comportamento, tanto do homem como da mulher, interferindo no ritmo de sua vida pessoal e conjugal. Neste sentindo, é de suma importância a orientação dos casais de meiaidade com a finalidade de auxiliá-los a compreenderem o processo do climatério e superarem as dificuldades que possam surgir. Da mesma forma é preciso educar o coletivo, que traz arraigados em si crenças e conceitos errôneos sobre a mulher climatérica para romper com a ideia de "finitude", incorporando a ideia de continuidade do processo de vida.

\subsection{Tratamentos utilizados para o climaté- rio}

Algumas participantes mencionaram a utilização de chás banho de acento com plantas medicinais no tratamento para o climatério, como descrito nos relatos a seguir:

Eu nunca usei nada, às vezes eu tomo uma casca de pau, que é bom pra limpar o corpo... quixabeira, cajueiro, aroeira e barbatimão é muito bom minha mãe usava e me ensinou usar também tomo a água e também faço o banho de acento ( TULIPA).

Tomo chá de amora miúra, muito bom, depois que comecei a tomar esse chá não tenho mais aqueles calores (ROSA).

A Organização Mundial de Saúde (OMS) define as plantas medicinais como espécies de vegetais que possuem em um de seus órgãos, ou em toda planta, substâncias que, se administradas ao ser humano ou a animais, por qualquer via e sob qualquer forma, exercem algum tipo de ação farmacológica (SOUSA et al., 2013).

As plantas medicinais podem ser consideradas um recurso para o auxílio do tratamento do climatério, então, os profissionais da saúde, em especial, os enfermeiros devem adquirir este conhecimento para transmitir à comunidade, conhecendo os benefícios e os riscos que cada planta medicinal pode influenciar no tratamento do paciente (SOUSA et al., 2013). 
É importante salientar que o uso da fitoterapia é mais uma opção terapêutica ofertada aos usuários do SUS (Sistema Único de Saúde), por meio do Decreto Federal de no 5.813 de 22 de junho de 2006, instituiu a Política Nacional de Plantas Medicinais e Fitoterápicos. Nesse sentido faz-se necessária capacitação dos profissionais de saúde quanto ao uso racional de plantas medicinais e de fitoterápicos (SOUSA et al., 2013).

Por outro lado, as mulheres não utilizaram nenhum tipo de medicação industrializada para o tratamento dos sintomas que relacionaram a fase do climatério. Esse período necessita uma atenção maior no seu método de tratamento, sendo indispensável a avaliação do risco e benefício do uso a curto ou a longo prazo da Terapia de Reposição Hormonal (TRH).

A reposição hormonal continua a apresentar importância na assistência da mulher climatérica e na pós-menopausa, muito embora alguns trabalhos tenham mostrado resultados com mais riscos que benefícios. Esses estudos serviram para mostrar a necessidade de maior critério na indicação da reposição hormonal, assim como necessidade de individualização das mulheres para a indicação da reposição hormonal, bem como avaliar qual o melhor esquema para cada mulher (FONSECA, 2001).

Com o surgimento de restrições do uso da TRH, houve interesse pelas terapias complementares entre estas terapias o uso de plantas medicinais que é um método utilizado para amenizar os sintomas de fogachos conforme citado pelas mulheres como o "chá de amora miúra".
3.4 Assistência de enfermagem à saúde da mulher durante o climatério

Nesta categoria as mulheres se expressaram da seguinte forma:

Nunca ouvir falar de climatério em lugar algum, nem aqui no posto, e eu frequento o posto há muito tempo, já passou um monte de enfermeira nenhuma falou sobre isso, aqui não tem muita palestra não pra gente... pode ser que agora mude é uma enfermeira nova [...] (JASMIM).

[...] faço o preventivo todo ano com a enfermeira, mas ela não fala disso não, era bom se ela falasse, tivesse palestra, assim a gente sabia mais das coisas... a gente passa por tanta coisa que a gente nem sabe, era bom pra gente que ela orientasse, que ela cuidasse mais da gente para não ter mais tanta dor[...] (LAVANDA).

[...] a assistência de enfermagem nesta fase não tem não a gente só faz o preventivo, mas ela não pergunta nada disso não, acho que a enfermeira devia cuidar mais da gente que tá nessa fase por que é difícil você passar por isso sozinha, a nossa saúde é um bem precioso e precisa de cuidado... o cuidado tem que melhorar se não como a gente fica [...](CAMÉLIA).

Os resultados dos depoimentos evidenciam que as mulheres não recebem a assistência de enfermagem adequada para esta fase da vida, que essa assistência deve ser melhorada como forma de obter maior qualidade assistencial. Perceber-se ainda, pelos depoimentos que o foco dos enfermeiros está na realização do Exame citopatológico de colo de útero. Observa-se que as mulheres sentem falta de atividades educativas como palestras, conversas e orientações do profissional enfermeiro. Além disso, mostram pouco conhecimento sobre as alterna- 
tivas terapêuticas para suas queixas, centrando somente o enfermeiro como a solução de seus problemas de saúde e de vida.

Para a Federação Brasileira de Ginecologia e Obstetrícia - FEBRASGO (PRAXEDES, 2011), é essencial que as mulheres que vivenciam o climatério tenham orientação de um profissional especializado que possam Ihe proporcionar uma assistência individualizada mais adequada, a fim de melhorar a qualidade de vida.

\section{CONCLUSÃO}

Neste trabalho, analisou-se a percepção de mulheres acerca das mudanças corporais e emocionais durante o climatério em uma Estratégia de Saúde da Família no município de Paulo Afonso, na Bahia. Foi possível constatar que o climatério é um período importante da vida da mulher, tanto quanto as demais fases de sua vida; entretanto, é visto como uma fase difícil.

Os resultados da pesquisa apontaram que a construção do saber destas mulheres sobre o climatério é vago, informal, passado através de gerações, muitas vezes vinculado a mitos e tabus. E, por isso, convivem com diversas dúvidas e anseios causando a elas uma apreensão quanto o período vivido. Sintomas decorrentes do climatério foram apontados como causa de desmotivação para o desempenho de qualquer atividade pessoal, prejudicando a qualidade de vida das mulheres, com reflexo no ambiente familiar, no trabalho na saúde e na promoção de seu bem-estar.

Constatou-se que as mulheres não fazem uso de terapia de reposição hormonal, nem medicamentos industrializados, mas que recorrem às plantas medicinais para tratar ou amenizar os sintomas referentes a esta fase. A sexualidade pode ser comprometida durante esse período, tanto por desordens físicas, biológicas, psicológicas quanto, até mesmo sociais. Há pouco auxílio assistencial da enfermagem às mulheres na vivência do climatério.

\section{REFERÊNCIAS}

ALMEIDA, A. B. de. Climatério. In: ALMEIDA, A. B. de (Org). Reavaliando o climatério: enfoque atual e multidisciplinar. São Paulo: Atheneu, 2003, p. 3-16.

ARAÚJO, I. A. As representações sociais da sexualidade/vida sexual da mulher no climatério: subsídios para o cuidado de enfermagem. Rio de Janeiro: UFRJ/EEAN, 2009.

BARDIN, L. Análise de conteúdo. 3 ed. Lisboa: Edições 70, 2004.

BIFFI, E. F. A. Saúde Mental e Climatério na Perspectiva de Mulheres. Profissionais de Saúde, Tese de Doutorado, 2003.

BRASIL. Ministério da Saúde. Secretaria de Atenção à Saúde. Departamento de Ações Programáticas Estratégicas. Manual de Atenção à Mulher no Climatério/Menopausa - Brasília: Editora do Ministério da Saúde, 2008.

CAMPANA, L. O. C.; NETO, A. M. P.; PEDRO, A. O. Conhecimento sobre a menopausa e seu tratamento de acordo com o estado menopausal e estrato social: análise de inquérito populacional domiciliar em muIheres climatéricas do município de campinas. Dissertação para obtenção de título de mestre. UNICAMP, 2001.

CAMPOS, H. H.; et al. Distúrbios do sono no climatério. Femina, vol. 33 , n. 11 , p. $815-$ 820, 2005.

CRUZ, C. S. Prevalência de sintomas climatéricos em mulheres na pré e transição 
menopáusica: estudo de base populacional. Dissertação de mestrado. UFRGS, 2009.

DE LORENZI, D. R. S; et al. Fatores indicadores da sintomatologia climatérica. Rev. Bras. Ginecol. Obstet, v. 27, n. 1, p. 12-19, 2005.

FONSECA, A. M.; et al. Menopausa e tabagismo. Rev. Ginec. Obstet, v. 10, n. 1, p. 2125, 1999.

FONSECA, A. M. Aspectos clínicos da reposição hormonal. Reprodução \& Climatério, v. 16, n. 2, p. 19-23, 2001.

FORTIN, M. Fundamentos e etapas do processo de investigação. Lisboa: Lusodidacta, 2009.

GONÇALVES, R.; MERIGHI, M. A. B. Climatério: novas abordagens para o cuidar. In FERNANDES, R. Á. Q; NARCHI, N. Z. Enfermagem e saúde da Mulher. Barueri, SP: Manole, 2007.

LIMA, J. V.; ANGELO, M. Vivenciando a inexorabilidade do tempo e as suas mudanças com perdas e possibilidades: a mulher na fase do climatério. Rev. Esc. Enferm. USP, v. 35, n. 4, p. 399-405, 2001.

MILANEZ, M. R. M.; NERY, I. S. Percepção das mulheres sobre o climatério: bases para a assistência de enfermagem. Escola Ana Nery Revista de Enfermagem, Rio de Janeiro, v. 8, n. 2, p. 199-204, ago. 2004.

MOURA, A. D. A.; et al. Conhecimento e motivação das mulheres acerca do exame de Papanicolau: subsídios para a prática de enfermagem. Revista RENE, v. 11, n. 1, p. 94-104, jan./mar. 2010.

PINTO, R. E. M. Avaliação da qualidade de vida no climatério entre usuárias e não usuárias de Terapia de Reposição Hormonal. Dissertação (Mestrado em Enfermagem). Fundação Universitária do Rio Grande/Goiás, 2006.
PRAXEDES, T. F. Percepção das mulheres em relação às mudanças corporais e emocionais no período do climatério. Monografia. UNESC, Campina Grande. 2011.

PRESTES, M. L. M. A pesquisa e a construção do conhecimento científico: do planejamento aos textos da escola à academia. 2 ed. São Paulo-SP: Réspel, 2003.

SANTOS, L. M. Síndrome do climatério e qualidade de vida: uma percepção das muIheres nessa fase da vida. Revista APS, v. 10, n. 1, p. 20-26, jan./jun. 2007.

SCLOWITZ, K. T. S.; SANTOS, I. S.; SILVEIRA, M. F. Prevalência e fatores associados a fogachos em mulheres climatéricas e pósclimatéricas. Caderno de Saúde Pública, Pelotas-RS, v. 21, n. 2, p. 469-481, mar/abr. 2005.

SOUSA, A. A; et al. Plantas medicinais em enfermagem e os saberes populares. Ribeira de Pombal: All Print Editora, 2013. SOUZA, L. M.; FIORAVANTE, E. Fatores associados à realização dos exames preventivos de câncer de mama e de colo uterino, pelas mulheres brasileiras. In: Encontro Nacional de Estudos Populacionais, Caxambu: CEDEPLAR, 2008. Disponível em: <http:www.a bep.nepo.unicamp.br/encontro2008/docsp df/abep2008_1211.pdf>. Acesso em: 01 nov. 2014.

VIDAL, C. R. P. M. Mulheres no climatério: desconhecimento, relacionamentos e estratégias. Dissertação (Mestrado Acadêmico em Cuidados Clínicos em Saúde). Universidade Estadual do Ceará, Centro de Ciências da Saúde. Fortaleza, 2009.

WANNMACHER, L; LUBIANCA, J. N. Terapia de reposição Hormonal na Menopausa: evidências atuais. Uso Racional de Medicamentos: temas selecionados, v. $1, \mathrm{n}$. 6, p. 1-6, 2004. 

access article distributed under the terms of the Creative Commons Attribution License, which permits unrestricted use, distribution, and reproduction in any medium, provided the original work is properly cited.

Artigo recebido em 02 de novembro de 2015.

Avaliado em 21 de março de 2016.

Aceito em 23 de maio de 2016.

Publicado em 25 de maio de 2016.

\section{Como citar este artigo (ABNT):}

SANTOS, Árquisa Antônia de Sousa; SILVA, Flávia Ventura da; MARTINS, Fabiana Lopes. Percepção das mulheres no município de Paulo Afonso, na Bahia, sobre as mudanças corporais e emocionais no período do climatério. Estação Científica (UNIFAP), Macapá, v. 6, n. 1, p. 91-104, jan./abr. 2016. 